\title{
Introductory Chapter: Understanding Wavelets
}

\author{
Sudhakar Radhakrishnan \\ Additional information is available at the end of the chapter \\ http://dx.doi.org/10.5772/intechopen.78388
}

\section{Introduction}

In this section, let us discuss some fundamentals which are required to understand wavelets. Signals which are coming from a source are normally in time domain. Examples are sinusoidal signal, bio-medical signal, etc. Anytime domain signal can be processed or transformed into frequency domain (spectral domain) using mathematical transformations. Fourier transform is one of the popular or famous transform that will convert a time domain signal into frequency domain signal without any loss of generality.

While plotting time domain signal, we use time in the x-axis and amplitude in the $y$-axis. The hidden information present in the signal cannot be revealed in the time domain hence a transform domain is required. The frequency content or spectrum of a signal is simply the frequency content (spectral components) of the signal. The frequency spectrum of a signal depicts what are all the frequencies exist in the signal. When plotting frequency domain, we use frequency in the $x$-axis and amplitude in the $y$-axis.

Normally for any signal, if the frequency content is not changing with respect to time is called as stationary signal. Example can be a sinusoidal signal where the frequency ' $\mathrm{X}$ ' $\mathrm{Hz}$ is not changing irrespective of the cycle. Unfortunately, real time signals are nonstationary signal where the frequency content of the signal is keeping on changing. The best example is biological signals. Suppose when we are looking at an ECG (electrocardiograph) signal. The typical shape of a healthy ECG signal is well known to cardiologists. Any significant deviation from that shape is usually considered to be a symptom of a pathological condition. Doctors analyse these cases not only in time domain, they are using frequency domain also to confirm the pathological condition. 


\section{Fourier transform (FT)}

To understand wavelets, let us go deep into the literature. The first and main contribution regarding the frequency content or spectrum came from the French mathematician John Baptise Fourier. He showed that any periodic function can be represented as an infinite sum of periodic complex exponential functions and named as Fourier transform (FT) [1].

Eqs. (1) and (2) represent the forward and inverse Fourier transform

$$
\begin{aligned}
& X(j \Omega)=\int_{-\infty}^{\infty} x(t) e^{-j \Omega t} d t \\
& x(t)=\int_{-\infty}^{\infty} X(j \Omega) e^{j \Omega t} d \Omega
\end{aligned}
$$

where $x(t)$ represents the time domain signal, $X(j \Omega)$ represents the frequency content of the signal and $\Omega=2 \times \pi \times \mathrm{F}$ and

$$
e^{j \Omega t}=\cos (\Omega t)+j \sin (\Omega t)
$$

Eq. (3) represents that any complex exponential is expressed as real part of cosine function and imaginary part of sine function with the corresponding frequency. As per Eq. (1), the input signal is multiplied with cosine function and sine function at all the time intervals and added (integrated) to yield the frequency content. The concept is best illustrated in Figures 1 and 2. Figure 1 shows the implementation at analysis side. Here, each blue coloured square is a narrow band pass filter with the cut off frequency $\Omega_{0^{\prime}} \Omega_{1^{\prime}} \ldots$.

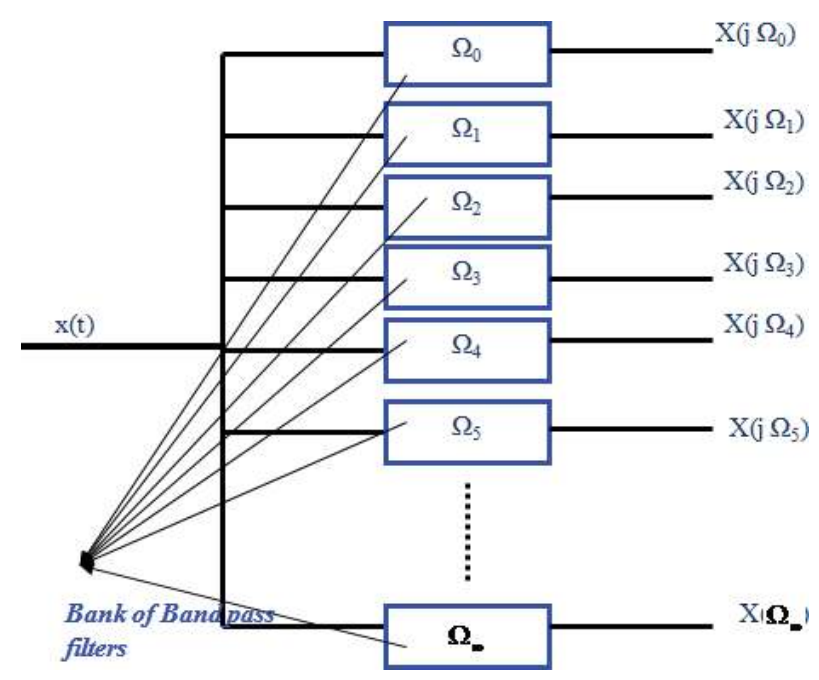

Figure 1. Fourier transform analysis side. 


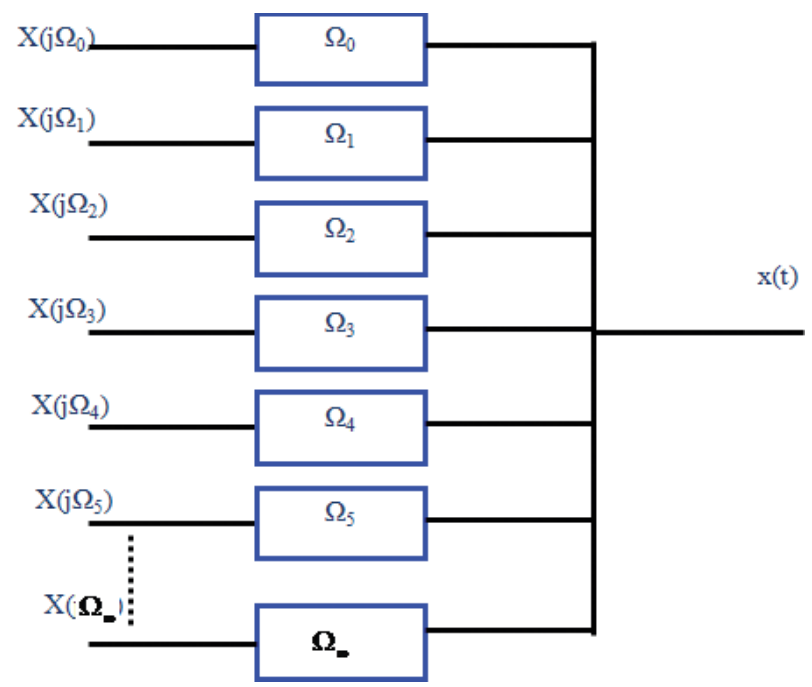

Figure 2. Fourier transform synthesis side.

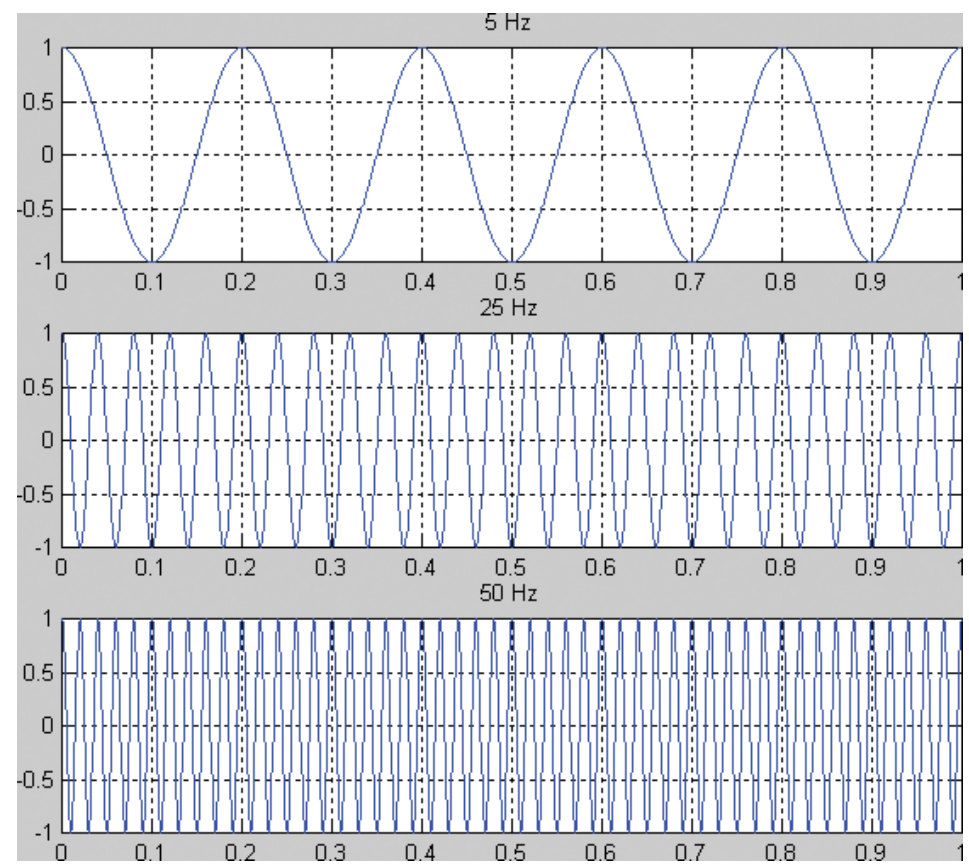

Figure 3. Cosine signal with three different frequencies 5, 25, and $50 \mathrm{~Hz}$. 

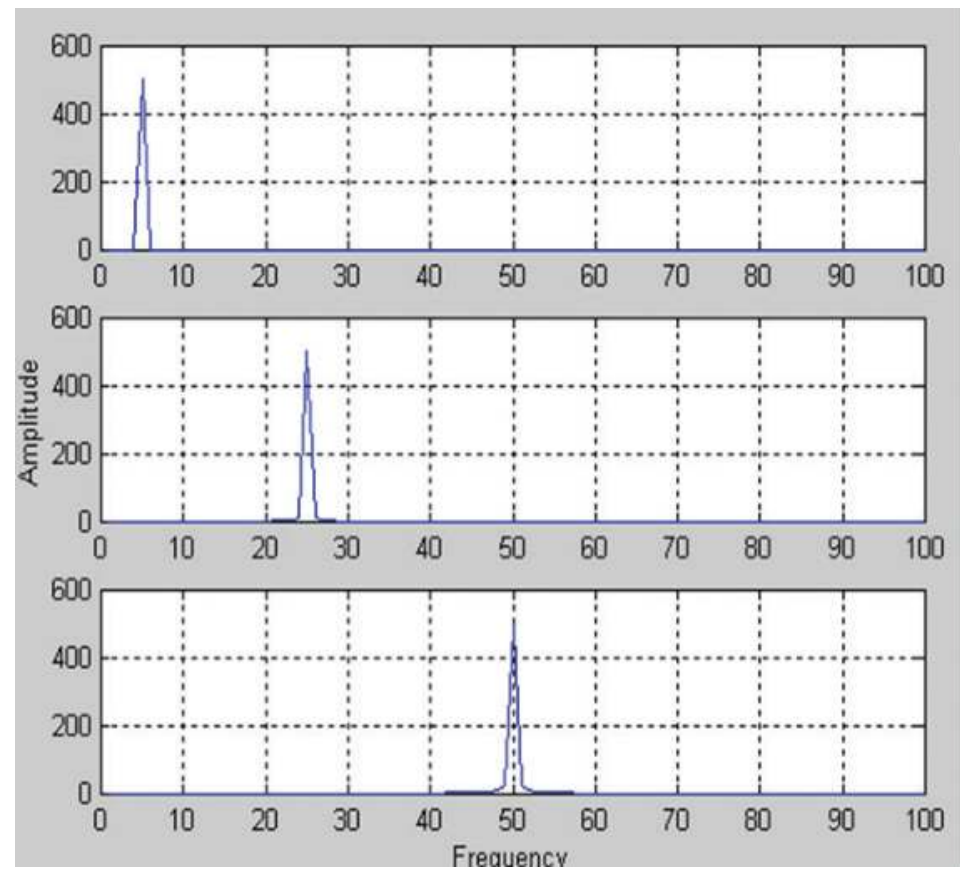

Figure 4. Spectrum cosine signal with three different frequencies 5, 25, and $50 \mathrm{~Hz}$.

Figure 2 shows the implementation at synthesis side. Here also, each blue coloured square is a narrow band pass filter with the cut off frequency $\Omega_{0}, \Omega_{1}, \ldots$

In Figure 1, if the result of the particular filter is large then, we can understand that the particular frequency component is dominant. If there is no output in any filter means that the particular frequency component is zero, i.e., single signal is passed multiple filter and the outputs are analysed and the reverse is happening in Figure $\mathbf{2}$ which is called as synthesis.

To understand the above one, see the following illustration shown in Figure 3 where the individual frequencies are indicated as 5,25 , and $50 \mathrm{~Hz}$. The corresponding spectrum is shown in Figure 4.

Figure 5 indicates a signal which contains all the three frequencies 5, 25, and $50 \mathrm{~Hz}$ mixed together and its corresponding spectrum.

Fourier transform is very much useful for a stationary signal. This means that Fourier transform clearly indicates what are all the frequency components exist in the given signal independent of time. Fourier transform completely fails for a nonstationary signal. 


$$
\begin{aligned}
x_{4}(t) & =\cos (2 \pi \cdot 5 \cdot t) \\
& +\cos (2 \pi \cdot 25 \cdot t) \\
& +\cos (2 \pi \cdot 50 \cdot t)
\end{aligned}
$$

$$
x_{4}(t) \stackrel{F}{\longrightarrow} X_{4}(\omega)
$$
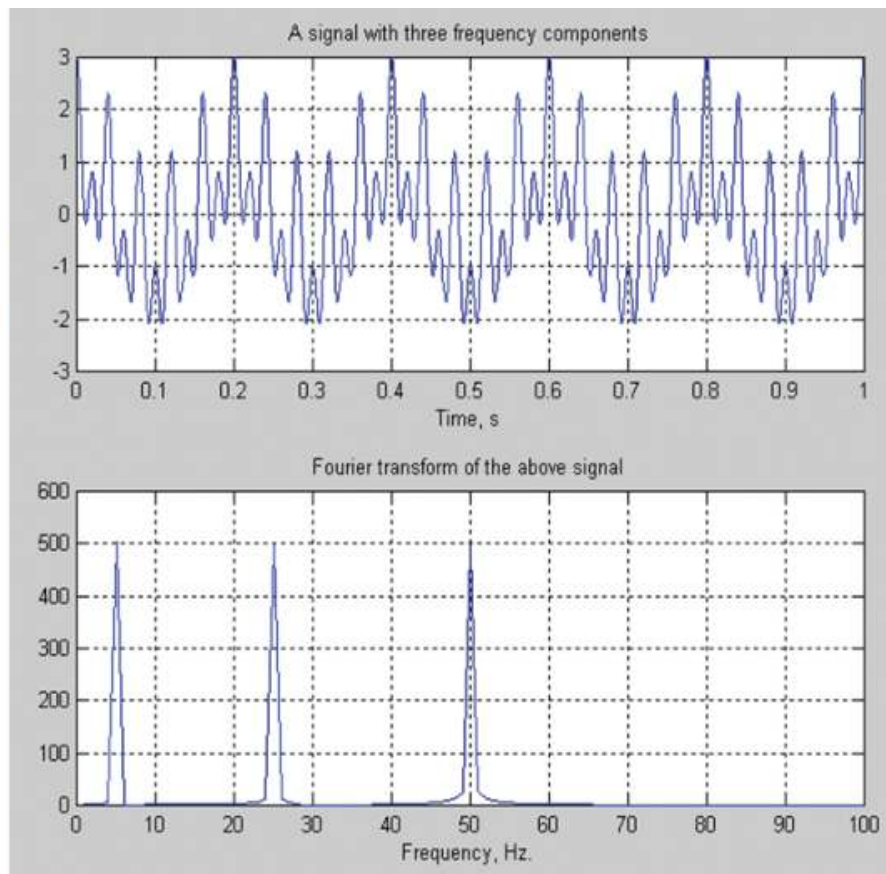

Figure 5. Cosine signal with three combined frequencies 5, 25, and $50 \mathrm{~Hz}$ and its corresponding spectrum.

\section{Short-time Fourier transform (STFT)}

As discussed previously that FT is not suitable for a nonstationary signal, a new set of transform is required which will provide timing and frequency information. The research moved forward and a conclusion was made that possibility of considering some portion of a nonstationary signal as stationary. This means that the long duration signal must be chopped for a short duration and possibility of finding the frequency components in that interval and this has to be completed for the entire signal to know the entire frequency components are present. The transform which provides this opportunity is short time Fourier transform (STFT). The STFT equation is given by

$$
\operatorname{STFT}_{x}^{W}(t, \Omega)=\int_{-\infty}^{\infty}[x(t) \times W(t-\tau)] e^{-j \Omega t} d t
$$

The above equation indicates that the input signal $x(t)$ is chopped by a window with a duration of ' $\tau$ ' and Fourier transform is taken. In other words, the signal is assumed to be stationary for the interval ' $\tau$ '. This process is repeated for the entire duration of signal. Now, somewhat the 


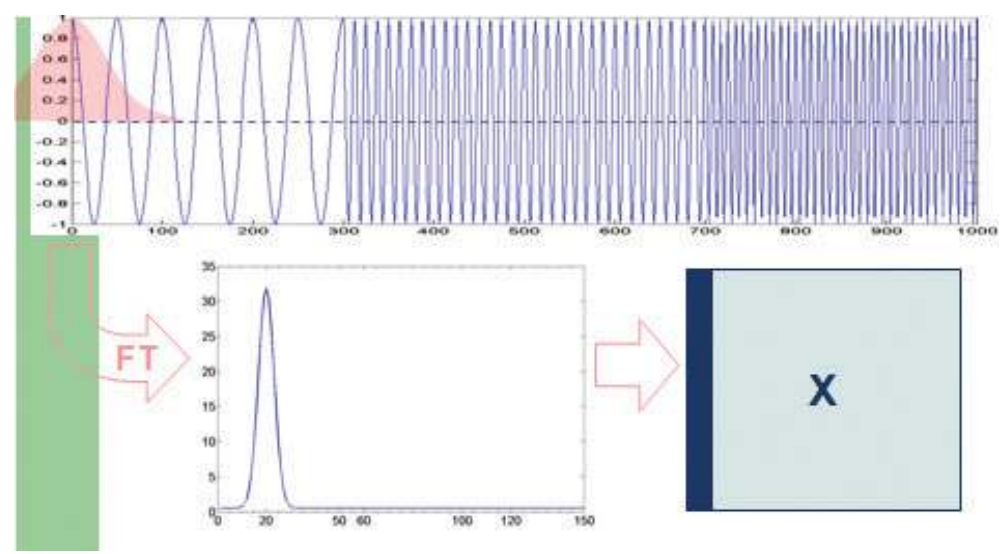

Figure 6. STFT of a nonstationary signal for one step.

problem faced by Fourier transform is solved and we get the frequency components with the window duration specified. Figure 6 indicates how STFT is taken for a nonstationary signal for one step of the window.

Now, the selection of the window width plays a vital role in STFT. The narrow window we select leads to poor frequency resolution and good time resolution which is shown in Figure 7. The opposite effect will happen if we select a wider window which is illustrated in Figure 8.
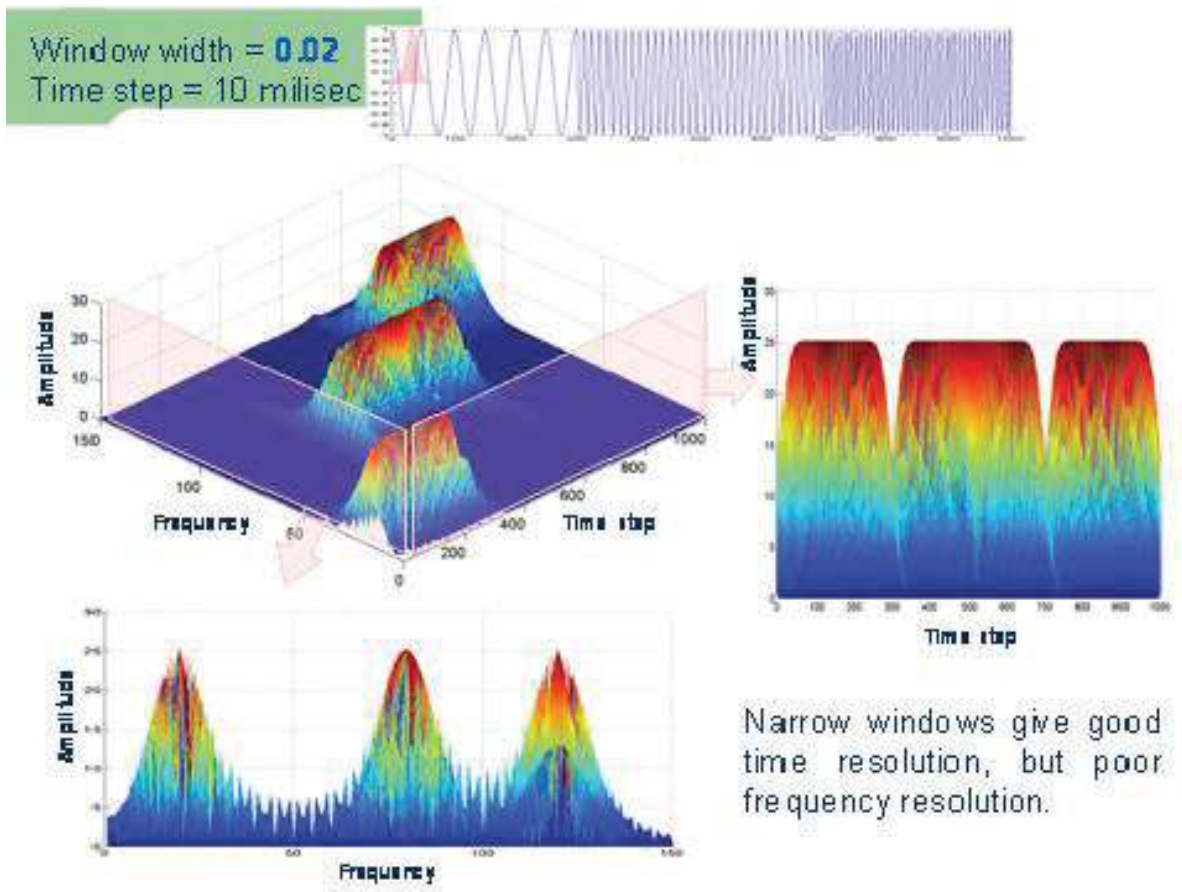

Narrow windows give good time resolution, but poor fre quency resolution.

Figure 7. Resolution for a narrower window. 


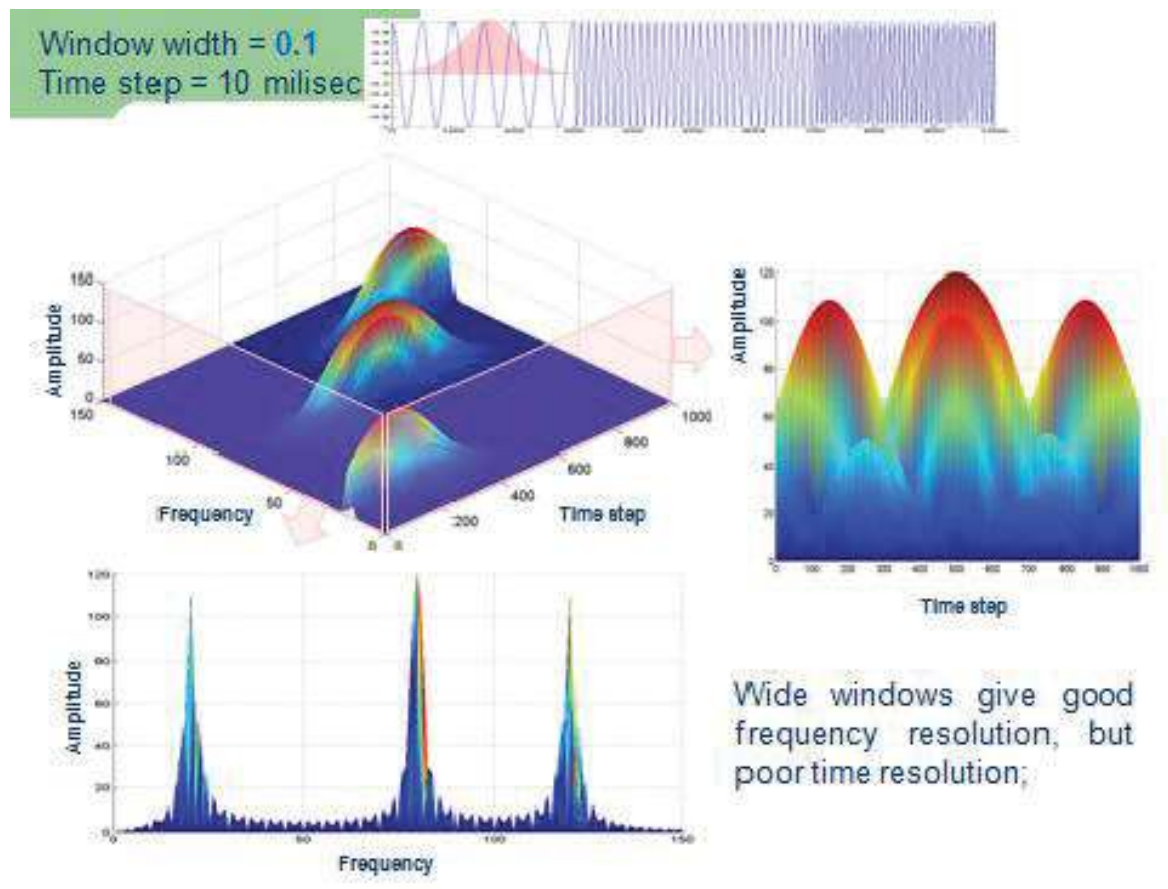

Figure 8. Resolution for a wider window.

Hence, the conclusion is STFT [1] that will provide a solution to the problem faced by Fourier transform but the drawback is there is a constant width of the window which is used and hence it provides only fixed resolution.

Normally, signal can carry both low frequency and high frequency components. To capture both, we need different widths of the window which is not provided by STFT. To understand more clearly, the concept of multiresolution is not there in STFT. Hence, we need a new transform which provides a solution to the above. The solution is wavelet transform.

\section{Continuous wavelet transform (CWT)}

The basic idea behind wavelet transform is, a new basis(window) function is introduced which can be enlarged or compressed to capture both low frequency and high frequency component of the signal (which relates to scale). The equation of wavelet transform $[2,3]$ is given in Eq. (5).

$$
W(a, b)=\frac{1}{\sqrt{|a|}} \int_{-\infty}^{\infty} x(t) \psi\left(\frac{t-b}{a}\right) d t
$$

where $W(a, b)$ is called the wavelet coefficient, ' $a$ ' is called the scaling parameter and ' $b$ ' is the shifting or translational parameter. $\psi(t)$ is called the mother wavelet. Different dilations and translations lead to different daughter wavelets. 
Any original data or signal can be represented in terms of a wavelet expansion. The best representation of a data using a wavelet depends on the best or close wavelet of what we are choosing. There are many numbers of wavelets available as per the literature. Some of the examples of the wavelets are Haar and Daubechies [3]; under Gaussian-based wavelets, we have Mexican hat wavelet and Morlet wavelet; under polynomial-based wavelets, we have Battle-lemarie, Coiflet and Spline-based wavelets; and under Sinc wavelets, we have Meyer wavelet and Shannon wavelet.

\section{Discrete wavelet transform (DWT)}

From the previous understanding, it is clear that CWT is a redundant transform, which means that the translation parameter ' $b$ ' and scaling parameter ' $a$ ' seem to be infinite making them difficult in terms of implementation. It is always seems to be CWT that is computable but not implementable. The solution for the implementation of wavelet transform arises from discrete wavelet transform (DWT). Sampling in the time-frequency plane on a dyadic (octave) grid is happening in DWT that makes them efficient in terms of implementation. The scaling parameter ' $a$ ' is replaced by $2^{-j}$ and ' $b$ ' is made proportional to ' $a$ ', i.e., $b=k 2^{-j}$. Here ' $j$ ' is called as scaling parameter and ' $k$ ' is the proportionality constant taking the role of shifting parameter in DWT. Substituting $a=2^{-j} ; b=2^{-j} k$ (j and k are integers) in Eq. (5), we get Eq. (6).

$$
\begin{aligned}
& \operatorname{CWT}_{x}^{\psi}(a, b)=\Psi_{x}^{\psi}(a, b)=\frac{1}{\sqrt{|a|}} \int_{t} x(t) \psi\left(\frac{t-b}{a}\right) d t \\
& \psi_{j, k}(t)=\sqrt{2^{j}} \psi\left(2^{j} t-k\right) j, k \in Z
\end{aligned}
$$

In multiresolution analysis, the signal can be viewed as the sum of a smooth ("coarse") part-reflects main features of the signal (approximation signal) and a detailed ("fine") part-faster fluctuations represent the details of the signal [1]. The separation of the signal

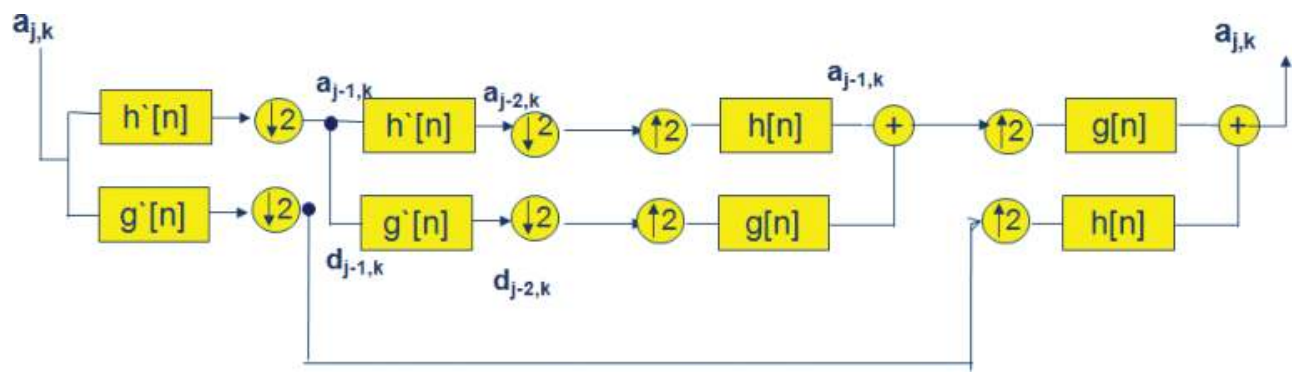

Figure 9. Filter bank implementation of DWT (courtesy by Robi polikar). 
into two parts is determined by the resolution given. This discussion introduces scaling function ' $\varphi_{i, k}(t)$ ' and wavelet function ' $\psi_{i, k}(t)$ ' and the corresponding approximation coefficients resulting from scaling function is denoted as $\mathrm{a}_{\mathrm{j}, \mathrm{k}}$ and the detailed coefficients resulting from wavelet function is denoted as $\mathrm{d}_{\mathrm{j}, \mathrm{k}}$. In implementation perspective, any signal is represented in Eq. (7).

$$
x(t)=\sum_{k=-\infty}^{\infty} a_{j_{0} k} \varphi_{j_{0} k}(t)+\sum_{j=-\infty}^{j_{0}} \sum_{k=-\infty}^{\infty} d_{j . k} \psi_{j, k}(t)
$$

Using Filter bank [2, 4], the implementation of Eq. (7) results in Figure 9, where h'[n] and g'[n] are the low pass and high pass filters in the analysis side and $h[n]$ and $g[n]$ are low pass and high pass filters in the synthesis side using the corresponding wavelet.

\section{Conclusion}

I hope that this chapter gives a definite and thoughtful introduction to all the beginners who are new to wavelets. As there are different number of wavelets available with different signal processing properties like compact support, symmetry, regularity and vanishing moments make them suitable in the field of signal de-noising, detecting discontinuities and breakdown points in a signal, compressing images, identifying pure frequencies, seismic and geophysical signal processing, video compression, acoustic data analysis, nuclear engineering, neurophysiology, music, magnetic resonance imaging, speech discrimination, optics, fractals, turbulence, earthquake-prediction, radar, human vision, etc. Some of applications from the perspective scientists and researchers are discussed in the forthcoming chapters.

\section{Author details}

Sudhakar Radhakrishnan

Address all correspondence to: sudha_radha2000@yahoo.co.in

Department of Electronics and Communication Engineering, Dr. Mahalingam College of Engineering and Technology, Pollachi, India

\section{References}

[1] The wavelet tutorial by Robipolikar. https://cseweb.ucsd.edu/ baden/Doc/wavelets/ polikar_wavelets.pdf 
[2] Vetterli M, Herley C. Wavelets and filter banks: Theory and design. IEEE Transactions on Signal Processing. 1992;40:2207-2232

[3] Daubechies I. Orthonormal bases of compactly supported wavelets. Communications on Pure and Applied Mathematics. 1988;41:906-966

[4] Abate A et al. Wavelets and Subbands Fundamentals and Applications. Newyork: Birkhäuser c/o Springer Verlag; 2002 